\title{
Changes in psychotropic prescription medication use and their relationship with mortality among people with traumatic spinal cord injury
}

\author{
Yue Cao ${ }^{1} \cdot$ Jillian M. R. Clark ${ }^{1} \cdot$ James S. Krause ${ }^{1}$
}

Received: 31 October 2017 / Revised: 2 February 2018 / Accepted: 2 February 2018 / Published online: 26 February 2018

( ) International Spinal Cord Society 2018

\begin{abstract}
Study design Prospective cohort study.

Objective To identify the relationship of frequency of prescription medication use to treat pain, spasticity, sleep, and depression/stress, and change in prescription medication use with risk of all-cause mortality.

Setting This study was initiated at a specialty hospital in the Southeastern USA, with follow-up and data analysis at an academic medical center in the Southeastern United States.

Methods Prospective data were collected in 1997-1998 (Time 1) and 2007-2009 (Time 2), with mortality determined as of 31 December 2014. The initial participant cohort was comprised of 1386 participants with traumatic SCI who were adults and a minimum of 1-year post-injury at enrollment in 1997-1998. Of these, 863 participated at follow-up and 861 were included in the current mortality analysis.

Results The frequency of taking prescription medication increased over the 10-year timeframe, particularly for sleep and pain. Each type of prescription medication at Time 1 was associated with later mortality, and an increase in medication use over time was associated with a greater risk of mortality for three of the four conditions (all except spasticity). A decrease in the frequency of pain medication use over time was associated with a decreased risk of all-cause mortality.

Conclusions More frequent use of prescription medication to treat pain, sleep, and depression/stress is related to all-cause mortality, and increases in the use of medications to treat these conditions is associated with elevated risk of all-cause mortality.
\end{abstract}

\section{Introduction}

Secondary health conditions occur frequently after spinal cord injury (SCI) and are associated with negative health, psychological, and social outcomes, as well as all-cause mortality [1-4]. These conditions are often managed pharmacologically, and individuals with SCI are frequently prescribed multiple medications. Recent research suggests individuals with SCI who use multiple prescribed medications are at higher risk for adverse drug events and medication errors [5, 6]. It is important to understand how

James S. Krause

krause@musc.edu

1 College of Health Professions, Medical University of South Carolina, Charleston, SC, USA prescription medication use impacts health and longevity in this population.

Evidence has begun to emerge identifying associations between prescribed medications and mortality in both specific health condition and community populations. For instance, among 5861 Danish patients with Parkinson's disease, of which $74 \%$ used at least one prescribed medication, Frandsen, Baandrup, Kjellberg, Ibsen, and Jennum [7] found utilization of an anti-depressant, benzodiazepinelike, and antipsychotic medication was associated with increased risk of all-cause mortality. In a study of stroke and matched controls, those using prescribed psychotropic medications within both populations showed increased risk of all-cause mortality [8]. Another study examining 21,826 community dwelling Norwegians suggested those who used any sleep medication had a two-fold increase in risk of allcause mortality when adjusting for physical and mental health and other medications [9]. This effect was higher for 
those using sleep medications daily and those prescribed benzodiazepines for sleep [9].

Limited research exists regarding the use of prescribed pain, sleep, antispasmodic, and other psychotropic medications as related to mortality in individuals with SCI, even though these medications are commonly used to treat secondary health conditions. In a study of 1209 participants with SCI first assessed in 1997-1998 and mortality identified in 2005, Krause, Zhai, Saunders, and Carter [10] determined that more frequent use of prescription medications for pain, spasticity, depression, and sleep was associated with higher risk of mortality. In an extension of this study using a larger participant cohort $(n=2535)$, the frequency of pain medication use was associated with a significant increase in mortality, above and beyond demographic, injury related, socioeconomic, and health characteristics [11]. Pain medication use had a stronger relationship with mortality than either pain intensity or pain interference, with those using pain medication daily being $51 \%$ more likely to be deceased despite the inclusion of pressure sores, amputation, hospitalization, and depression as risk factors. To our knowledge, there are no studies assessing the relationship between changes in prescription medication use and mortality after traumatic SCI.

\section{Purpose}

Our purpose was to identify the relationship of the frequency of prescription medication use and change in frequency of prescription medication use with all-cause mortality among 861 participants with traumatic SCI. We defined prescription medication use as frequency of use of prescription medication to treat pain, sleep, spasticity, and depression/stress. The frequency of prescription medication use was identified at two points in time over an approximate 10 -year interval. Prescription medication use relates to both health care provider practice and individual behavior, each of which are potential targets for intervention strategies to reduce excess mortality.

\section{Hypotheses}

1. Daily utilization of prescription medication to treat each of the four conditions will be associated with a greater risk of all-cause mortality.

2. An increase in the frequency of prescription medication use for each condition will be associated with a greater risk of all-cause mortality.

3. A decrease in the frequency of prescription medication use for each condition will be associated with a lower risk of all-cause mortality.

\section{Methods}

\section{Participants}

This is a prospective cohort study initiated in 1997-1998 (Time 1). Institutional Review Board approval was obtained before study initiation. Potential participants were identified from the SCI Model Systems patient database, registry, and outpatient directory at a rehabilitation specialty hospital in the southeastern USA. The inclusion criteria were: (a) minimum of 18 years old at time of measurement, (b) at least 1-year post-injury, and (c) residual effects resulting from traumatic SCI (American Spinal Injury Association Impairment Scale [AIS] A-D). Of a potential 1839 prospective participants, there were 1386 respondents at Time 1. Of these, 863 again participated in the second data collection in 2007-2009 (Time 2), 75 participants were lost, 119 no response, and 329 deceased between the two time points. Two participants were eliminated for missing key information, reducing the final sample to 861 participants.

\section{Data collection procedures}

There were two times of data collection, each of which used similar data collection procedures. Cover letters describing the study were sent approximately 4 weeks prior to mailing the first self-report assessment (SRA) questionnaire. Nonrespondents were sent a second mailing within 2 months of the initial mailing. Attempts were then made to contact nonrespondents by phone, if possible, and when requested, an additional packet of materials was sent. At Time 1, participants were offered $\$ 20$ in remuneration and made eligible for drawings totaling $\$ 1500$. Participants received $\$ 50$ in remuneration at Time 2 .

Mortality status was determined using the National Death Index (NDI), the gold standard for determining mortality. All records were sent to the NDI and mortality status was determined as of 31 December 2014. There were 157 deceased cases at follow-up.

\section{Measures}

The predictor of interest was frequency of use of prescription medications to treat four conditions: pain, spasticity, sleep, and depression/stress. Participants were asked to identify the frequency with which they took medications to treat each condition, with four options (never, rarely, weekly, daily). The items were phrased "over the past year how often did you use prescription drugs (those prescribed by your doctor) to treat ... [pain, spasticity, sleep, depression/stress]". These items have been previously used in mortality analyses, with a single point in time measurement $[10,12]$. In addition to identifying prescription medication 
use at each time of measurement, we identified medication change over the 10-year period (from Time 1 to Time 2), classified into one of three categories: (1) less frequent use of medication-self-report usage frequency at Time 2 was less than that at Time 1 (e.g., a change from weekly use to only occasional use); (2) no change in frequency-selfreport usage frequency at Time 2 was the same as that at Time 1; (3) more frequent use of medication-self-report usage frequency at Time 2 was more than that at Time 1 (e.g., a change from weekly to daily use).

We measured five demographic covariates at Time 1: age at the measurement, gender, race/ethnicity, low household income, and low education. Race/ethnicity was dichotomized into non-Hispanic white and other racial/ethnic minorities. Annual household income less than $\$ 20,000$ was defined as low income, and years of education less than 12 was defined as low education. We also measured injury severity by using four categories: C1-C4 level injury and non-ambulatory, C5-C8 level injury and non-ambulatory, non-cervical injury and non-ambulatory, and ambulatory. This classification of injury severity follows that reported in the literature, with ambulatory status a proxy measure for the AIS $[13,14]$. We measured self-rated general health by using a 5 -point scale $(1=$ poor health, $5=$ excellent health) [15], one of the most widely used health indicators in population surveys [16]. Although some studies suggest it is a conservative measure of health $[17,18]$, it is generally regarded as reliable and valid in self-assessment research [16, 19-22]. Prior research has supported a strong association between self-rated health and mortality across different race and ethnic groups in the United States [23]. For the current study, response options were combined into the following categories: very good/excellent health, good health, and poor/fair health.

\section{Analysis}

All statistical analyses used SAS 9.4. We first conducted descriptive analysis on participants' survival status, age, gender, race/ethnicity, injury severity, household income, education, and self-rated general health. We then calculated the percentages of three change categories for all four prescription drugs among all participants and among different gender and racial/ethnic groups. The Chi-square test was used to indicate whether the bivariate comparisons were statistically significant.

We developed four Cox proportional hazards models for multivariate analyses, with the survival days between the SRA date and the death date (or 31 December 2014 for those alive) as the dependent variable. All four Cox models had the same covariates: age, gender, race/ethnicity, low household income, low education, and general health. Each model also included Time 1 usage of the respective medication as an additional covariate. Due to the categories of "sometimes" and "weekly" usage having low frequency, these were combined in the covariate. The variables of interest in the Cox models were: (1) spasticity medication change, (2) pain medication change, (3) sleep medication change, and (4) depression/stress medication change, respectively. We needed to use separate models for each predictor because of the relatively low sample size with longitudinal data. Hazard ratios (HR) and $95 \%$ confidence intervals are reported for each variable in the model.

\section{Statement of ethics}

We certify that all applicable institutional and governmental regulations concerning the ethical use of human volunteers were followed during the course of this research.

\section{Results}

Among 861 participants, 72\% were male, 73\% nonHispanic white, $12 \% \mathrm{C} 1-\mathrm{C} 4$ level injury and non-ambulatory, 30\% C5-C8 level injury and non-ambulatory, 34\% non-cervical injury and non-ambulatory, and 24\% ambulatory. Approximately $46 \%$ of participants had low household income and $16 \%$ had low education. The average age at Time 1 was $38.8(\mathrm{SD}=11.8)$, and the average general health score was $3.3(\mathrm{SD}=1.0)$. The average interval between the first participation and follow-up was 10.4 years $(\mathrm{SD}=6.3)$. The average interval between the follow-up measurement and a determination of mortality status was 6.4 years $(\mathrm{SD}=1.7)$. At the end of the study, 157 participants $(18 \%)$ were deceased.

\section{Changes in medication use}

The stability of medication use varied considerably between the four medications (Table 1), with the most significant increases for pain medication (35\% increase compared with $15 \%$ decrease), followed by sleep medication (29\% increase, $13 \%$ decrease). Less substantial increases were

Table 1 Medication change from Time 1 to Time 2 among all participants

\begin{tabular}{llll}
\hline Medication & Less usage & $\begin{array}{l}\text { No change } \\
n \text { (row \%) }\end{array}$ & More usage \\
\hline Spasticity & $106(13.04)$ & $589(72.45)$ & $118(14.51)$ \\
Pain & $121(14.60)$ & $417(50.30)$ & $291(35.10)$ \\
Sleeping & $103(12.62)$ & $477(58.46)$ & $236(28.92)$ \\
Depression/stress & $87(10.60)$ & $572(69.67)$ & $162(19.73)$ \\
\hline
\end{tabular}


Table 2 Medication changes from Time 1 to Time 2 among different groups

\begin{tabular}{|c|c|c|c|c|c|c|c|c|c|}
\hline \multirow[t]{3}{*}{ Medication } & \multicolumn{3}{|l|}{ Gender } & \multicolumn{3}{|c|}{ Race/ethnicity } & \multicolumn{3}{|c|}{ Survival status } \\
\hline & Female & Male & $p$ & Minorities & $\begin{array}{l}\text { Non-Hispanic } \\
\text { White }\end{array}$ & $p$ & Survived & Deceased & $p$ \\
\hline & \multicolumn{3}{|c|}{ Column (\%) } & \multicolumn{3}{|c|}{ Column (\%) } & \multicolumn{3}{|c|}{ Column (\%) } \\
\hline Spasticity & & & 0.64 & & & $<0.01$ & & & 0.73 \\
\hline Less usage & 13.10 & 13.01 & & 16.11 & 11.98 & & 13.43 & 11.19 & \\
\hline No change & 74.24 & 71.75 & & 62.09 & 76.21 & & 71.94 & 74.83 & \\
\hline More usage & 12.66 & 15.24 & & 21.80 & 11.81 & & 14.63 & 13.99 & \\
\hline Pain & & & 0.25 & & & 0.37 & & & $<0.01$ \\
\hline Less usage & 17.80 & 13.32 & & 17.43 & 13.61 & & 16.57 & 5.44 & \\
\hline No change & 49.15 & 50.76 & & 49.54 & 50.66 & & 50.59 & 48.98 & \\
\hline More usage & 33.05 & 35.92 & & 33.03 & 35.74 & & 32.84 & 45.58 & \\
\hline Sleeping & & & 0.07 & & & 0.01 & & & 0.04 \\
\hline Less usage & 16.31 & 11.15 & & 15.17 & 11.75 & & 13.08 & 10.49 & \\
\hline No change & 53.22 & 60.55 & & 63.51 & 56.62 & & 59.88 & 51.75 & \\
\hline More usage & 30.47 & 28.30 & & 21.33 & 31.62 & & 27.04 & 37.76 & \\
\hline $\begin{array}{l}\text { Depression/ } \\
\text { stress }\end{array}$ & & & 0.47 & & & 0.47 & & & 0.03 \\
\hline Less usage & 11.59 & 10.20 & & 12.80 & 9.85 & & 10.06 & 13.10 & \\
\hline No change & 66.52 & 70.92 & & 67.30 & 70.44 & & 71.60 & 60.69 & \\
\hline More usage & 21.89 & 18.88 & & 19.91 & 19.70 & & 18.34 & 26.21 & \\
\hline
\end{tabular}

noted for depression/stress medication (20\% increase, $11 \%$ decrease). The least amount of change was for spasticity medication (15\% increase, $13 \%$ decrease).

Participants who were racial/ethnic minorities were more likely to increase their spasticity medication usage, while those who were non-Hispanic white were more likely to increase their sleep medication. Gender was unrelated to change in usage. Those who survived to follow-up were less likely to have increased their usage of pain medication, sleep medication, and depression/stress medication (Table 2).

\section{Predictive models}

All four Cox models (Table 3) indicated higher mortality was significantly associated with older age, being male, greater severity of injury, and low income. Lower self-rated general health was significantly associated with higher mortality in three models, except for pain medications.

The frequency of medication use was significant in each of the four models. The strongest relationships with mortality were for sleep and pain medication. Compared with those who did not use medication at the Time 1, participants who reported using sleep medication daily were $236 \%$ (HR $=3.36$ ) more likely to be deceased at follow-up. Similarly, participants using pain medication daily were $229 \%$ (HR = 3.29) more likely to be deceased at follow-up. Use of depression/stress medication and spasticity medication daily were also significant, with $109 \%(\mathrm{HR}=2.09)$ and $78 \%$ $(\mathrm{HR}=1.78)$ greater hazard of mortality, respectively.

After controlling for all the covariates, including Time 1 medication use, those who had an increased frequency of pain medication use during the 10 years were $94 \%$ ( $\mathrm{HR}=$ 1.94) more likely to be deceased compared to those who did not change medication usage; those who increased the frequency of sleep medication use were $81 \%(\mathrm{HR}=1.81)$ more likely to be deceased; and those who increased depression/stress medication use were 67\% $(\mathrm{HR}=1.67)$ more likely to be deceased. Participants who decreased the frequency of their pain medication usage were $69 \%$ (HR = 0.31) less likely to be deceased at the end of study (Table 3). Change in spasticity medication was not significantly related to mortality.

\section{Discussion}

To our knowledge, this is the first study investigating changes in the frequency of prescription medication use and all-cause mortality. By focusing on prescription medication use, the study lays the foundation for prevention strategies that target both the prescribing and utilization of prescription medication to treat multiple conditions. It also opens the door to search for discovery of new interventions, or the application of other proven interventions, to treat pain, sleep, spasticity, and depression/stress. 
Table 3 Cox proportional hazards models for predicting mortality after SCI

\begin{tabular}{|c|c|c|c|c|}
\hline & $\begin{array}{l}\text { Spasticity medication } \\
n=777 \\
\text { HR }(95 \% \text { CI })\end{array}$ & $\begin{array}{l}\text { Pain medication } \\
n=790 \\
\text { HR }(95 \% \text { CI })\end{array}$ & $\begin{array}{l}\text { Sleeping medication } \\
n=777 \\
\text { HR }(95 \% \mathrm{CI})\end{array}$ & $\begin{array}{l}\text { Depression/stress medication } \\
n=783 \\
\text { HR }(95 \% \mathrm{CI})\end{array}$ \\
\hline Age & $1.07(1.05-1.08)$ & $1.07(1.05-1.08)$ & $1.07(1.06-1.08)$ & $1.07(1.05-1.08)$ \\
\hline Male (ref: female) & $1.70(1.11-2.62)$ & $1.86(1.21-2.85)$ & $1.70(1.11-2.59)$ & $1.63(1.08-2.48)$ \\
\hline Non-Hispanic White (ref: others) & $1.47(0.96-2.25)$ & $1.28(0.84-1.94)$ & $1.29(0.84-2.00)$ & $1.28(0.83-1.97)$ \\
\hline \multicolumn{5}{|l|}{ Injury severity (ref: all ambulatory) } \\
\hline $\mathrm{C} 1-4$, non-ambulatory & $4.69(2.43-9.06)$ & $4.68(2.52-8.71)$ & $6.07(3.18-11.56)$ & $5.67(3.01-10.67)$ \\
\hline C5-8, non-ambulatory & $3.11(1.74-5.57)$ & $3.20(1.85-5.51)$ & $3.25(1.84-5.74)$ & $3.28(1.87-5.75)$ \\
\hline Non-cervical, non-ambulatory & $2.18(1.22-3.91)$ & $1.77(1.02-3.07)$ & $2.32(1.30-4.12)$ & $2.06(1.17-3.64)$ \\
\hline Low income (ref: income $\geq 20 \mathrm{~K}$ ) & $1.76(1.21-2.57)$ & $1.64(1.12-2.40)$ & $1.76(1.21-2.57)$ & $1.86(1.28-2.70)$ \\
\hline Low education (ref: education years $\geq 12$ ) & $1.08(0.70-1.67)$ & $0.93(0.61-1.41)$ & $1.00(0.65-1.56)$ & $1.01(0.65-1.55)$ \\
\hline \multicolumn{5}{|c|}{ Self-rated general health (ref: very good/excellent health) } \\
\hline Poor/fair health & $2.05(1.27-3.29)$ & $1.54(0.96-2.46)$ & $1.73(1.07-2.80)$ & $1.77(1.11-2.83)$ \\
\hline Good health & $1.37(0.90-2.07)$ & $1.35(0.90-2.04)$ & $1.32(0.87-2.00)$ & $1.27(0.84-1.91)$ \\
\hline \multicolumn{5}{|l|}{ Baseline medication usage (ref: never) } \\
\hline Sometimes/weekly & $1.87(0.94-3.71)$ & $1.30(0.85-1.99)$ & $0.93(0.54-1.61)$ & $0.56(0.25-1.30)$ \\
\hline Daily & $1.78(1.15-2.74)$ & $3.29(1.99-5.46)$ & $3.36(2.03-5.56)$ & $2.09(1.27-3.43)$ \\
\hline \multicolumn{5}{|l|}{ Medication usage change (ref: no change) } \\
\hline More usage & $0.98(0.53-1.81)$ & $1.94(1.26-2.98)$ & $1.81(1.21-2.72)$ & $1.67(1.09-2.56)$ \\
\hline Less usage & $0.60(0.33-1.09)$ & $0.31(0.14-0.70)$ & $0.64(0.34-1.19)$ & $1.03(0.55-1.94)$ \\
\hline
\end{tabular}

The findings support the first hypothesis, as each of the four types of daily medication use were significantly related to higher all-cause mortality, whereas the second hypothesis was partially supported. While worse self-rated general health was found to be associated with increased risk of mortality in all but the pain medication model, increases in medication use from Time 1 to Time 2 for most medications (all except spasticity) were associated with increased risk of mortality when controlling for self-rated health. The third hypothesis was only supported for pain medication use, as decreasing use was associated with diminished all-cause mortality.

Consistent with initial research highlighting relationships between psychotropic [7, 8] sleep [9], and pain medications [11] and mortality, the findings expand our knowledge of these relationships in individuals with traumatic SCI. In the general population, death related to accidental overdose on opioid medications has been identified as an increasing problem [24, 25]. Adverse effects such as over sedation, respiratory depression, immune suppression, and impaired balance may be potential mechanisms in which psychotropic, sleep, and pain medications are associated with mortality and secondary health conditions [26].

The current results raise concerns over the use of prescription medications by suggesting associations between use and increased use over time and increased risk of allcause mortality. Although they should not be taken as direct evidence that the prescription medication use causes mortality per se, given that underlying conditions no doubt affect mortality, at a minimum, the identified associations do support the necessary consideration of changes in prescription medication use as a potential marker for risk of mortality.

\section{Clinical implications}

Although the findings cannot be unequivocally interpreted to indicate a direct cause of medication use or change in medication use and all-cause mortality, they support the need for caution in prescribing medications and further understanding of the long-term consequences of these medications on health and longevity. Non-pharmacological treatments are needed wherever possible to improve outcomes. Prescribing medications is easy and perhaps less costly in terms of time but does raise the concern of adverse consequence. Individuals with traumatic SCI also need to consider carefully the decision as to whether to utilize medication to treat particularly pain, sleep, and depression/ stress, as well as increasing the frequency of use of these medications. It is essential that healthcare professionals consider potential complications and share this information with those with traumatic SCI so they can make informed decisions. Exploration of alternative evidence supported treatments, such as psychotherapeutic approaches for pain, sleep disturbance, and psychological distress, among others, should be considered. 


\section{Strengths and limitations}

There are several distinctive strengths. First, there is an absence of investigations of risk factors for all-cause mortality related to prescription medication use. Although it may be confounded with other factors related to health, prescription medication use is a behavior that can be changed at both a system (prescribing) and individual level. Second, we identified actual changes in prescription use behaviors and risk of mortality. Therefore, it may serve as a guide for individuals who take or are considering using medications to treat secondary conditions or symptoms of traumatic SCI. It helps them and healthcare professionals to understand the association of prescription medication use and change in medication use over time with mortality.

In terms of limitations, all data on health status and prescription medication use are self-report. Therefore, there may be some random errors in reporting which would make it more difficult to identify truly significant differences, such that we may underestimate the strength of the relationship between prescription medication use and mortality. Second, there is a time lag in the recording of deaths within the NDI, so it is possible or even likely that some deaths were missed or misclassified as alive. Although we feel this is relatively limited, it would again limit the strength to identify the relationships between prescription medication use and mortality. Third, for a prospective cohort study of mortality, the sample size is relatively small. This is problematic for less frequently reported prescription medication use (i.e., occasional, weekly), rather than the larger sample for those who either do not use medication at all or do so daily. It also limits our ability to test multiple types of medication use within the same model (the reason for using four separate models). Fourth, this is a study of all-cause mortality, so, while establishing relationships between the frequency of medication change and all-cause mortality is the first step, ultimately, studies will be needed that address causespecific mortality. Lastly, our information on prescription medication use is general. We only know the frequency of use based on four categories. We cannot differentiate between dosages, more frequent use within the categories, or, most importantly, type of medications.

\section{Future research}

There are clear needs for further research. First, we need investigations that detail the types, dosages, and detailed frequency of medications used among people with SCI. We also need to understand how these types of usage patterns relate to multiple outcomes, not just mortality. The literature is clearly limited in studies relating medication use, or medication misuse, with SCI outcomes. Further, it is important to augment both the sample size and the number of times of measurement, so we may better understand how medication use changes over time and how it affects health outcomes. These design enhancements would also allow for inclusion of multiple medications within the same model, along with additional controls for comorbid health conditions. Third, research is needed that directly investigates causes of death related to unintentional overdose, as this would evaluate the direct link between prescription medication and causes of death. Such research would supplement that from the current study that establishes the more general association, potentially contribution, of medication use and all-cause mortality. Lastly, as always, we need to develop and test intervention strategies to reduce adverse outcomes associated with prescription medication use.

\section{Conclusion}

Increased utilization of prescription medication to treat pain, sleep, and depression/stress is associated with increased risk of all-cause mortality.

Acknowledgements The contents of this publication were developed under grants from the National Institute on Disability, Independent Living, and Rehabilitation Research (NIDILRR grant number 90IF0066, 90RT5003, and 90SI5016). NIDILRR is a Center within the Administration for Community Living (ACL), Department of Health and Human Services (HHS). The contents of this publication do not necessarily represent the policy of NIDILRR, ACL, HHS, and you should not assume endorsement by the Federal Government.

\section{Compliance with ethical standards}

Conflict of interest The authors declare that they have no conflict of interest.

\section{References}

1. Krause JS, Saunders LL. Health, secondary conditions, and life expectancy after spinal cord injury. Arch Phys Med Rehabil. 2011;92:1770-5.

2. McColl MA, Arnold R, Charlifue S, Glass C, Savic G, Frankel H. Aging, spinal cord injury, and quality of life: structural relationships. Arch Phys Med Rehabil. 2003;84:1137-44.

3. Jensen MP, Kuehn CM, Amtmann D, Cardenas DD. Symptom burden in persons with spinal cord injury. Arch Phys Med Rehabil. 2007;88:638-45.

4. Meade MA, Barrett K, Ellenbogen PS, Jackson MN. Work intensity and variations in health and personal characteristics of individuals with spinal cord injury. $J$ Vocat Rehabil. 2006;25:13-9.

5. Kitzman P, Cecil D, Kolpek JH. The risks of polypharmacy following spinal cord injury. J Spinal Cord Med. 2017;40:147-53.

6. Patel T, Milligan J, Lee J. Medication-related problems in individuals with spinal cord injury in a primary care-based clinic. $\mathrm{J}$ Spinal Cord Med. 2017;40:54-61.

7. Frandsen R, Baandrup L, Kjellberg J, Ibsen R, Jennum P. Increased all-cause mortality with psychotropic medication in 
Parkinson's disease and controls: a national register-based study. Park Relat Disord. 2014;20:1124-8.

8. Jennum P, Baandrup L, Iversen HK, Ibsen R, Kjellberg J. Mortality and use of psychotropic medication in patients with stroke: a population-wide, register-based study. BMJ Open. 2016;6: e010662.

9. Sivertsen B, Madsen IEH, Salo P, Tell GS, Øverland S. Use of sleep medications and mortality: the Hordaland Health Study. Drugs Real World Outcomes. 2015;2:123-8.

10. Krause JS, Zhai Y, Saunders LL, Carter RE. Risk of mortality after spinal cord injury: an 8-year prospective study. Arch Phys Med Rehabil. 2009;90:1708-15.

11. Krause JS, Cao Y, Clark JMR. Pain intensity, interference, and medication use after spinal cord injury: relationship with risk of mortality after controlling for socioeconomic and other health factors. Arch Phys Med Rehabil. 2017; 98(12):2464-70.

12. Krause JS, Carter RE, Pickelsimer E. Behavioral risk factors of mortality after spinal cord injury. Arch Phys Med Rehabil. 2009;90:95-101.

13. Saunders LL, Krause JS, Acuna J. Association of race, socioeconomic status, and health care access with pressure ulcers after spinal cord injury. Arch Phys Med Rehabil. 2012;93:972-7.

14. Saunders LL, Krause JS. Behavioral factors related to fatigue among persons with spinal cord injury. Arch Phys Med Rehabil. 2012;93:313-8.

15. CDC. Behavioral risk factor surveillance system survey questionnaire. Atlanta, GA: US Department of Health and Human Services, Centers for Disease Control and Prevention; 2006 [cited 2009 July 16]. http://www.cdc.gov/brfss/questionnaires/pdf-ques/ 2006brfss.pdf.

16. Idler EL, Benyamini Y. Self-rated health and mortality: a review of twenty-seven community studies. J Health Soc Behav. 1997;38:21-37.
17. George LK, Bearon LB. Quality of life in older persons: meaning and measurement. New York: Human Sciences Press; 1980. xii, $238 \mathrm{pp}$.

18. Maddox GL. Self-assessment of health status: a longituinal study of selected elderly subjects. J Chron Dis. 1964;17:449-60.

19. Farmer MM, Ferraro KF. Distress and perceived health: mechanisms of health decline. J Health Soc Behav. 1997;38:298-311.

20. Hays JC, Schoenfeld D, Blazer DG, Gold DT. Global self-ratings of health and mortality: hazard in the North Carolina Piedmont. J Clin Epidemiol. 1996;49:969-79.

21. Johnson RJ, Wolinsky FD. The structure of health status among older adults: disease, disability, functional limitation, and perceived health. J Health Soc Behav. 1993;34:105-21.

22. Lynch SM. Cohort and life-course patterns in the relationship between education and health: a hierarchical approach. Demography. 2003;40:309-31.

23. McGee DL, Liao Y, Cao G, Cooper RS. Self-reported health status and mortality in a multiethnic US cohort. Am J Epidemiol. 1999;149:41-6.

24. Paulozzi LJ, Jones CM, Mack KA, Rudd RA. Vital signs: overdoses of prescription opioid pain relievers-United States, 19992008. Morb Mortal Wkly Rep. 2011;60:1477-509.

25. US Department of Health and Human Services. Addressing prescription drug abuse in the United States: current activities and future opportunities. Washington, DC: US Department of Health and Human Services; 2013.

26. Geller AI, Nopkhun W, Dows-Martinez MN, Strasser DC. Polypharmacy and the role of physical medicine and rehabilitation. Phys Med Rehabil. 2012;4:198-219. 